\title{
Nuevas aportaciones a la obra del arquitecto Luis Ferreres Soler. El proyecto del Mercado de Cullera (Valencia)
}

\author{
Adrià Besó Ros \\ Universitat de València \\ adria.beso@uv.es
}

RESUMEN: El mercado de Cullera, proyectado en 1894 por el arquitecto Luis Ferreres, se concluye en 1902. Plantea una propuesta caracterizada por su singularidad tipológica, que enlaza con otros destacados proyectos y realizaciones, donde confluyen las experiencias de su autor en los ámbitos de la arquitectura y del urbanismo. El estudio de su detallada memoria explicativa, hasta ahora inédita, nos permite entenderlo como respuesta a las necesidades particulares del lugar. Analizamos su emplazamiento y su relación con el entorno urbano, el programa de necesidades a los que pretende dar respuesta y por último abordamos la propuesta arquitectónica, caracterizada por la sencillez y racionalidad asociada al tipo. Todo ello nos permite valorar este mercado como una obra coherente con la trayectoria profesional de su autor y situarlo como uno de los más destacados que se construyen en el ámbito valenciano fuera de la capital.

PALABRAS CLAVE: Arquitectura industrial; Mercado de abastos; Obra pública; Historia del urbanismo; Arquitectura del hierro; Luis Ferreres; Valencia.

\section{New Contributions to the Architect Luis Ferreres Soler Work. Cullera Market Project (Valencia)}

ABSTRACT: The Cullera market, designed in 1894 by the architect Luis Ferreres, was completed in 1902. It formulates a proposal characterized by its typological uniqueness, which links with other outstanding projects and achievements, where its author's experiences in architecture and urban planning areas converge. The study of its hitherto unpublished detailed explanatory memorandum, allows us to understand it as a response to the particular needs of the place. We analyse its location and its relationship with the urban environment, the programme of needs to which it intends to respond and finally we approach the architectural proposal, characterised by the simplicity and rationality associated with the type. All this allows us to assess this market as a work coherent with the professional career of its creator, making it one of the most outstanding buildings outside the capital city of Valencia.

KEYWORDS: Industrial architecture; Foodstuffs market; Public works; History of town planning; Iron architecture; Luis Ferreres; Valencia.

Recibido: 28 de febrero de 2018 / Aceptado: 7 de enero de 2019.

\section{Introducción}

Durante el último tercio del siglo XIX se manifiesta en muchas ciudades un notable interés por ordenar y regular el comercio al por mayor y al detall a partir de la construcción de unas instalaciones estables. De esta manera, en muchas capitales y poblaciones que gozaron de cierto dinamismo económico como consecuencia de su desarrollo industrial o agrícola, las corporaciones municipales plantearon la construcción de nuevos mercados con la finalidad de mejorar la higiene y el ornato público, de acuerdo con una nueva imagen urbana de progreso y modernidad. El auge de la agricultura comercial experimentado en las comarcas litorales del golfo de Valencia hizo que, entre finales del siglo XIX y primeras décadas del siglo XX, muchas poblaciones se dotaran de modernos mercados para adaptar su imagen urbana a los niveles de riqueza adquiri-

Cómo citar este artículo: BESÓ ROS, Adrià, «Nuevas aportaciones a la obra del arquitecto Luis Ferreres Soler. El proyecto del Mercado de Cullera (Valencia)», Boletín de Arte-UMA, n. ${ }^{\circ}$ 40, Departamento de Historia del Arte, Universidad de Málaga, 2019, pp. 267-279, ISSN: 0211-8483, e-ISSN: 2695-415X, DOI: http://dx.doi.org/10.24310/ BoLArte.2019.v0i40.5657 
dos. En este sentido, el desarrollo económico alcanzado en la comarca de la Ribera gracias a la especialización en la naranja en las tierras altas y en el arroz en los marjales litorales, donde se encuentra Cullera, posibilitó una temprana modernización de la imagen urbana de muchas poblaciones, que vino acompañada de mejoras en el ornato público y de la construcción de nuevos equipamientos para el abasto. Dentro de este contexto podemos situar el nuevo mercado de Cullera, proyectado en julio de 1894 por el entonces arquitecto municipal Luis Ferreres. Tras salir a licitación pública ${ }^{1}$ las obras fueron adjudicadas el 7 de noviembre de 1899 por un importe de 131.168,69 ptas. El proceso de construcción finalizó el 3 de diciembre de 1902 con la recepción definitiva de la tercera sección².

Luis Ferreres Soler (1852-1926) obtuvo el título de arquitecto en la escuela de Madrid en 1876. Durante los primeros años trabajo como ayudante de su maestro, Francisco Jareño, en las obras del edificio de la Biblioteca Nacional en el paseo de Recoletos de Madrid. En 1883 obtuvo la plaza de arquitecto de distrito en el Ayuntamiento de Valencia, a la que renunció con posterioridad para participar en diversos concursos y obras municipales. Entre ellas destacamos el primer plan definitivo de ensanche, que redactó junto con José Calvo y Joaquín M. a Arnau en 1887. En 1888 obtuvo el primer premio para la construcción del nuevo mercado central con un proyecto presentado en colaboración con Adolfo Morales, que no llegó a ejecutarse, cuyo documento no se ha conservado (Benito, 1992: 346-350; Taberner, 1988; 2009: 132-134). En 1898 se aprobó la construcción del matadero municipal de Valencia, inaugurado en 1901, que es su obra pública más conocida ${ }^{3}$. En 1899 proyectó una nueva galería para el Museo de Bellas Artes de la Real Academia de San Carlos. Compaginó estos grandes trabajos en la capital con otras obras de arquitectura y urbanismo en varias ciudades valencianas. Es autor de los planes de ensanche de Gandia (1880), Algemesí (1893) y Cullera (1896). Proyectó diversos mataderos y mercados. Entre los segundos destacan los de Algemesí (20-10-1894), Cullera (26-7-1894), Moncada (1905) y Silla (8-7-1907)4. Como explica Francisco Taberner, la discreción entendida como virtud fue el rasgo que definió la personalidad y la trayectoria profesional de Ferreres. Por ello,

[... en su arquitectura se advierte un diseño exento de alardes y cuidadosa elaboración, una composición rotunda y decidida, un estudiado repertorio ornamental de arraigadas raíces clasicistas y un profundo conocimiento de la técnica constructiva que distingue su obra y la convierte en una importante aportación a la arquitectura valenciana de este período. Una arquitectura que refleja la personalidad de un arquitecto que pese a su talante discreto, propició sin embargo audaces y renovadoras propuestas (Taberner, 2009: 144).

Esta discreción, unido al carácter funcional de estos equipamientos colectivos que ha motivado que hasta hace unas décadas no se hayan reconocido como portadores de valores culturales y estéticos, ha hecho que este conjunto haya pasado prácticamente desapercibido hasta la fecha, como buena parte de su obra. En este estudio se pretende dar a conocer este proyecto y situar el mercado de Cullera como uno de los más destacados de la Comunidad Valenciana por su singularidad (Taberner, 2009: 134). A partir del análisis de su detallada memoria podemos ver como no se queda únicamente en el diseño arquitectónico, sino que además se plantea como una propuesta urbanística, pues realiza un estudio para determinar su localización más adecuada, contempla las necesidades actuales y futuras y proyecta un conjunto de cuatro pabellones a partir de la ordenación del espacio público que conforma una gran plaza arbolada.

\section{La elección del emplazamiento y su relación con la ciudad}

El núcleo urbano de Cullera se asienta entre el piedemonte de la muela del Castillo y el cauce del Júcar, ya cerca de su desembocadura. Como consecuencia de los ataques de piratas berberiscos, en 1551 se autorizó la construcción de un nuevo recinto amurallado que garantizara la seguridad de sus habitantes ante nuevas invasiones, que con pequeñas reparaciones y modificaciones, permaneció hasta la segunda mitad del siglo XIX. Su derribo comenzó con el torreón del Río en 1861 y se completó en 1868 (Arciniega, 2002: 244). El caserío se había ido expandiendo fuera del recinto amurallado en dirección norte, sobre las laderas de la montaña, y hacia el este. La desaparición de la muralla posibilitó la redacción de un plan de ensanche por el mismo Ferreres en 1896, que permitió ordenar el crecimiento que se programaría sobre el llano existente hacia el este, en dirección al mar5. 
1. Francisco Roglá López, Mercado municipal de Cullera, 1925, vista del pabellón de la sección primera, con la subida al santuario al fondo, Biblioteca Valenciana Nicolau Primitiu. Fons Roglá

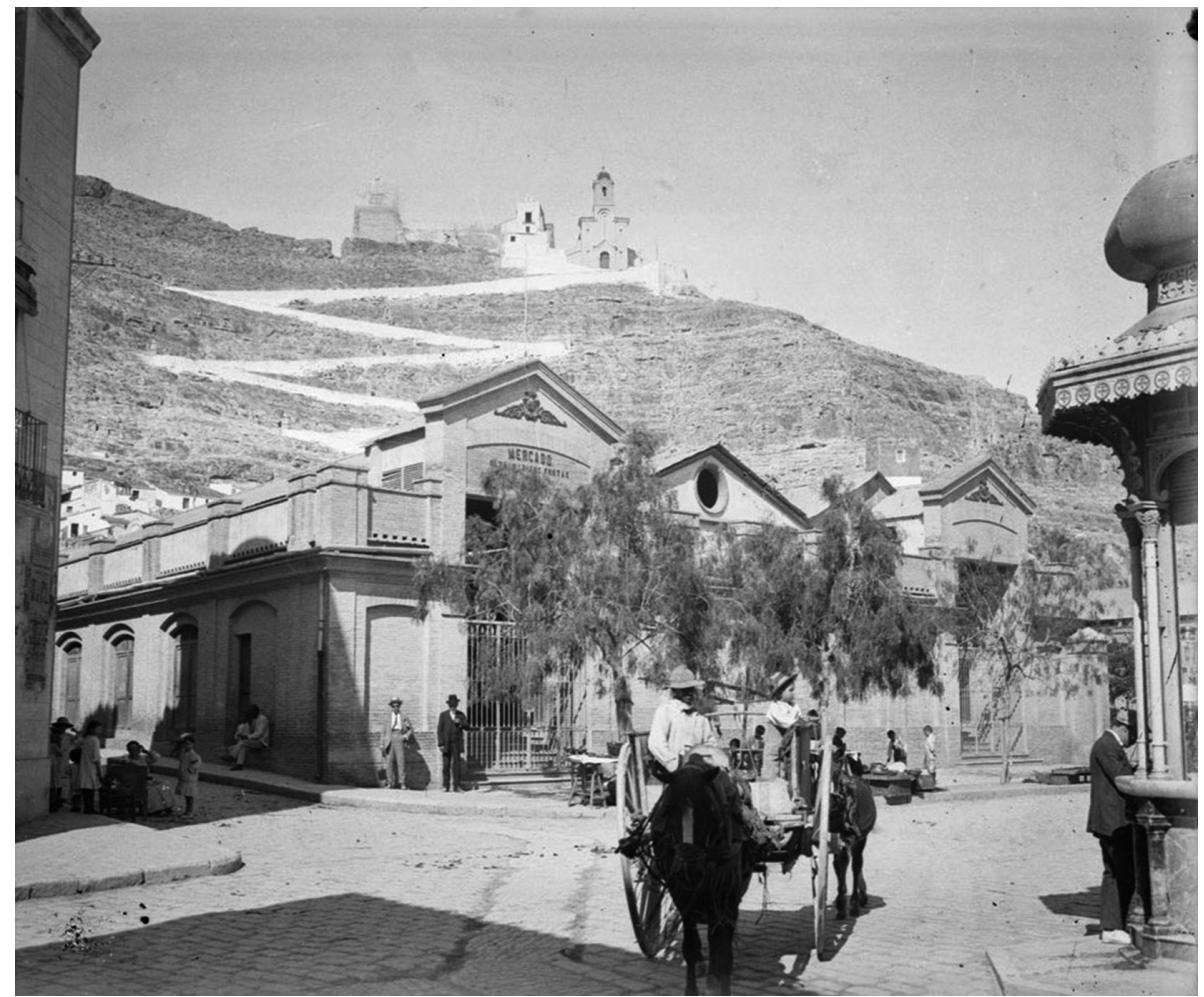

La memoria del proyecto se inicia con un estudio del mercado existente, de sus características y sus limitaciones para una población que sobrepasaba entonces los doce mil habitantes. Sus puestos se repartían entre las plazas de la Reina y de la Constitución, de pequeñas dimensiones, y la calle de la Cisterna (actuales plazas de la Verdura, de España y calle del Mestre Valls respectivamente), causando numerosas molestias y falta de higiene. Para su ubicación propone como emplazamiento más adecuado una explanada de forma trapezoidal, ocupada por varias eras y secaderos de arroz, situada entre el antiguo perímetro amurallado y la zona hacia donde había comenzado a desarrollarse el caserío extramuros y se orientaba el crecimiento previsto por el plan de ensanche. Ferreres plantea diversos argumentos para justificar su propuesta.

Por un lado, la morfología de la trama urbana y la dificultad de accesos impedían una continuidad con la localización histórica. Tampoco encontró dentro de los límites del antiguo perímetro amurallado ningún espacio abierto adecuado. Además de estos condicionantes propios del lugar, Ferreres no era partidario de construir los nuevos mercados sobre los antiguos emplazamientos como también explica en la memoria del proyecto de Algemesí (Besó, 2017: 363365), criterio se mantiene de forma constante en la ubicación de todos los mercados que proyecta.

La previsión de buenos accesos era importante para el adecuado funcionamiento, pues facilitaban la llegada de mercancías y a su vez la afluencia de compradores (Giralt, 1937: 22). En este sentido la parcela elegida para su construcción recaía a la calle del Mar, una de las principales vías que articulaban el crecimiento del ensanche y a su vez de acceso al centro histórico. Su posición más o menos centrada respecto al conjunto del núcleo urbano favorecía una buena accesibilidad y servicio a todo el vecindario.

La ausencia de edificaciones facilitaba la adquisición de este terreno por parte del Ayuntamiento. Uno de los principales problemas que se plantearon en la construcción de mercados fue el de las expropiaciones. Teniendo en cuenta las limitaciones presupuestarias que tenían en aquel momento las haciendas municipales, las dificultades de hacer frente al pago de los justiprecios en muchos casos ocasionaron demoras importantes en su construcción, como ha- 


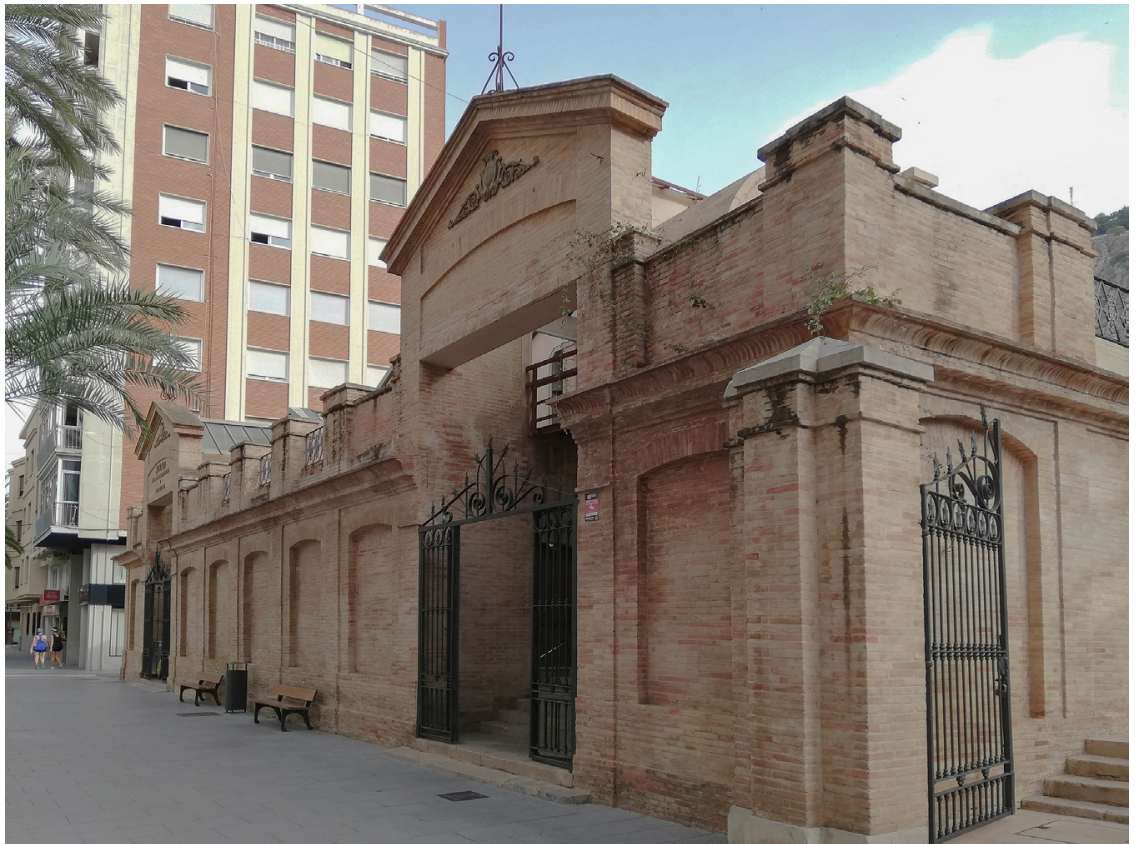

2. Mercado Municipal de Cullera, 2019, imagen actual del pabellón de la sección primera bía ocurrido en la vecina localidad de Sueca ${ }^{6}$, o incluso unos años más tarde en la construcción del Mercado Central de Valencia (Hidalgo, 2012).

\section{El programa de necesidades como punto de partida}

A la hora de abordar esta cuestión imprescindible en cualquier proyecto, Ferreres definió una serie de necesidades específicas relacionadas con los usos ocasionales del emplazamiento, con la situación actual del comercio local y con las propias de la ejecución material del conjunto, sin dejar de lado las que debía cumplir cualquier mercado moderno.

Las eras donde se proponía levantar el mercado eran punto de encuentro de la población para la peregrinación al santuario de la Virgen del Castillo el día de su festividad, celebrada el 8 de septiembre, pues esta explanada se sitúa a los pies de la subida al calvario por la que se accedía [1], [2]. Ferreres cree conveniente que se continúe manteniendo este uso como punto de reunión, por lo que plantea que el nuevo mercado no debe ocupar toda la superficie disponible. Por ello traza una avenida de $30 \mathrm{~m}$ de ancho que enlaza la calle del Mar con la subida al Castillo, que quedaría atravesada en perpendicular por otra de 20 m, lo que le permitía plantear una división del proyecto en cuatro secciones. Además, estos espacios libres también podían servir para ubicar el mercado semanal ambulante al aire libre sin causar molestias en las calles adyacentes. De esta forma confluyen dentro del mismo perímetro el mercado permanente, que se desarrollaría a diario bajo los pabellones de las cuatro secciones, y el ambulante de periodicidad semanal, cuyos puestos se emplazarían en las avenidas interiores sin necesidad de interferir en el las diversas funciones urbanas desarrolladas en la vía pública.

Al programa de necesidades que debe satisfacer un mercado moderno, añade las específicas de la población de Cullera, que identifica a partir de un estudio del mercado que se venía celebrando al aire libre. Los puestos fijos destinados a carnicerías y ultramarinos se situaban en los bajos de las casas de las calles y plazas donde se desarrollaba la actividad comercial. A ellas acudían también los cosecheros locales a vender sus productos de temporada, por lo que no necesitaban de unos puestos permanentes. Según los datos facilitados por el Ayuntamiento, en ese momento participaban 306 vendedores, los cuales se concentraban mayoritariamente durante el verano, especialmente en la venta de melones y sandías. En base a esta información propone que el nuevo mercado debe disponer de un número deter- 


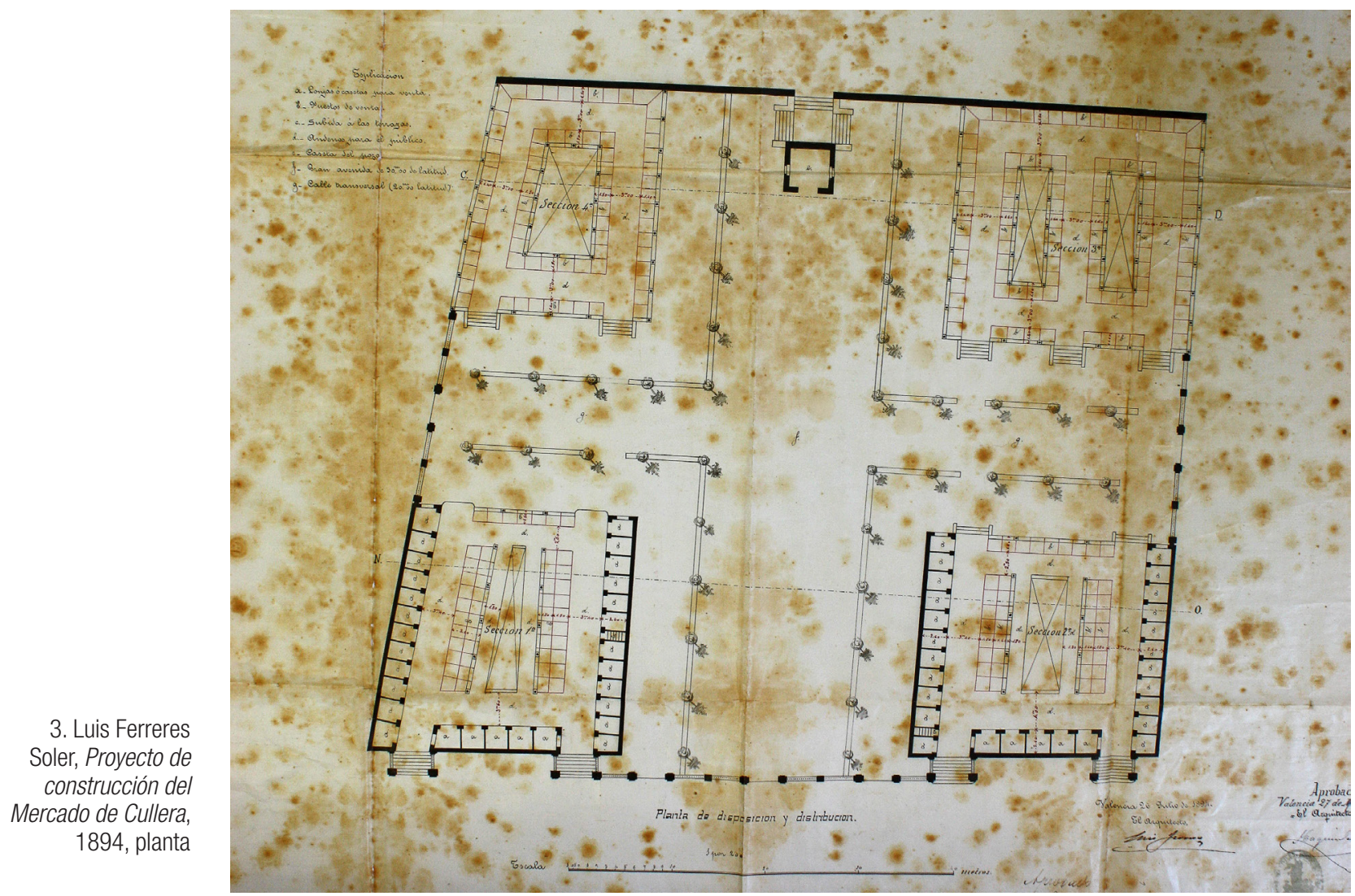

minado de puestos fijos en forma de casetas o lonjas cerradas para instalar carnicerías y tiendas de ultramarinos; un número suficiente de puestos fijos permanentes a cubierto para fruterías y verdulerías; y dejar grandes superficies de reserva para establecer otros puestos estacionales para la venta de sandías y melones durante el verano, que podrían estar al exterior por la ausencia de lluvias.

Otros condicionantes guardan relación con las características topográficas del emplazamiento, que por su desnivel obligaba a realizar operaciones de desmonte. Al situarse al pie de la montaña del Castillo recoge parte de sus escorrentías. Pero en este caso, por su escaso caudal, propone el desvío de las aguas en superficie por las calles laterales resultantes y no ve la necesidad de construir una canalización subterránea como hizo en el mercado de Silla (Ferrer, 1997: 158).

Fracciona el proyecto para permitir su ejecución en cinco fases o secciones independientes para que esta manera resulte lo menos gravosa para las arcas municipales.
Como explica Ferreres, es más conveniente realizarlo por secciones completas, que no plantear un proyecto unitario y construirlo por partes, ya que suele producirse un desfase entre lo proyectado y lo construido, además de las consiguientes molestias que causa a sus usuarios mientras se prolongan las obras ${ }^{7}$. La primera fase o sección en ejecutarse sería la de obras generales, que contempla el acondicionamiento del terreno con los desmontes y nivelaciones, construcción de muros de contención del cerramiento perimetral. Y luego cada una de las cuatro secciones o pabellones de que constaría el mercado, que podían construirse por separado. Estas estarán especializadas en diferentes tipos de productos: carnes de todas clases; ultramarinos y similares; pescados, salazones, ranas y caracoles; legumbres hortalizas, destinando los puestos centrales a la venta de frutas y verduras, sin perjuicio de que en verano ocupen además otros espacios al aire libre por la mayor oferta [3].

Esta solución tipológica carece de referentes en el ámbito valenciano. El modelo que Víctor Baltard planteó para 


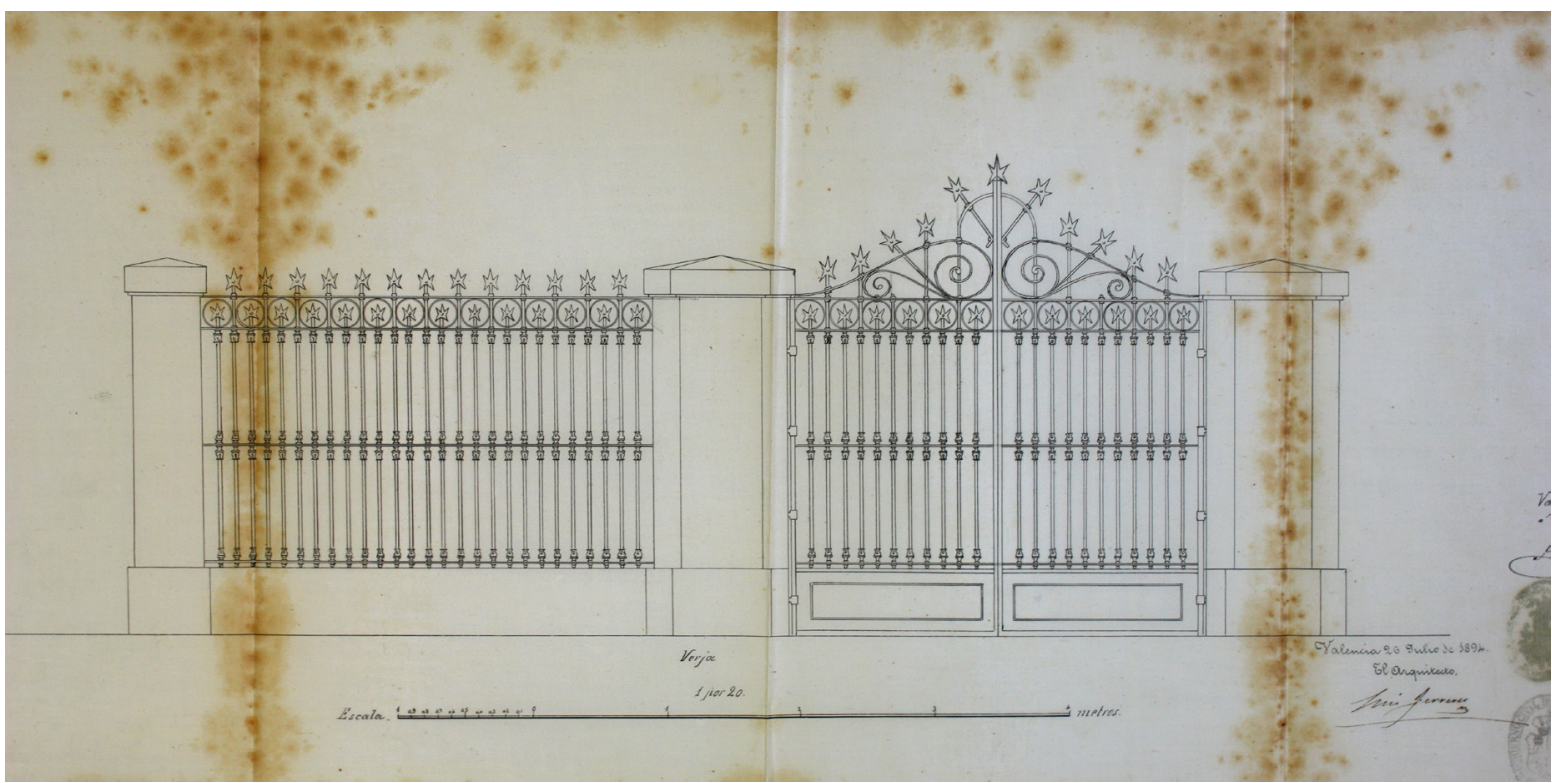

4. Luis Ferreres Soler, Proyecto de construcción del Mercado de Cullera, 1894, detalle del cerramiento exterior

las Halles Centrales de París (1854-66) formadas por diversos pabellones organizados a partir de una estructura de galerías cubiertas, tuvo su repercusión en España entre otros, en los madrileños mercados de Mostenses (1867-75) o de San Miguel (1915-1916) cuyos alzados se articulan a partir de la combinación de ejes longitudinales y perpendiculares cubiertos, entre los cuales se levantaban los diferentes pabellones. Entre ellas encontramos también referentes de mercados con cuatro secciones formadas por dos pasajes perpendiculares cubiertos (Castañer, 2006: 195-197), como los proyectos no realizados para los mercados de la Plaza de Bilbao en Madrid (1867, Mariano Calvo Pereira) o el de la Barceloneta en Barcelona (1873, Antonio Rovira y Trías). Posiblemente Ferreres tuvo en cuenta este concepto, aunque lo adapta a las necesidades concretas que plantea en el proyecto, y obtiene como resultado esta obra singular.

Por último analiza las ventajas de la construcción del mercado, que se pueden resumir en las tres que indica Pelayo Clairac (1887, V: 294): higiene, facilidad en la circulación y comodidad para vendedores y compradores. $Y$ de funcionalidad urbana, pues la ejecución de este proyecto supondrá la descongestión de la zona donde se ubica el Ayuntamiento, que es donde se localizaban los puestos de venta en aquel momento.

\section{Definición del proyecto arquitectónico}

La parcela donde se proyecta la construcción del mercado tiene forma de paralelogramo y dispone de una superficie de $5.304 \mathrm{~m}^{2}$. En los espacios resultantes por el cruce de estas dos avenidas se levantarán los pabellones que conforman las cuatro secciones, especializadas en diferentes productos. Para disminuir los efectos del calor durante el verano y aumentar la comodidad de los usuarios prevé la plantación de árboles en estas calles. El espacio situado en la vía transversal entre las secciones anteriores y posteriores se concibe a su vez como una reserva de suelo en previsión de futuras necesidades de ampliación para poder construir naves abiertas ${ }^{8}$.

Para acondicionar un terreno en desnivel, Ferreres plantea mantener la pendiente de la avenida longitudinal hasta su cruce con la calle transversal. Esta cota serviría de referencia para las plataformas sobre la que se edificarían los cuatro pabellones. El desnivel resultante al final de la avenida longitudinal se salvaría con unas escaleras para posibilitar el acceso a la subida al Castillo.

El perímetro del mercado queda totalmente cerrado por los muros de los pabellones que conforman las diferentes secciones y en el espacio de separación que queda en- 


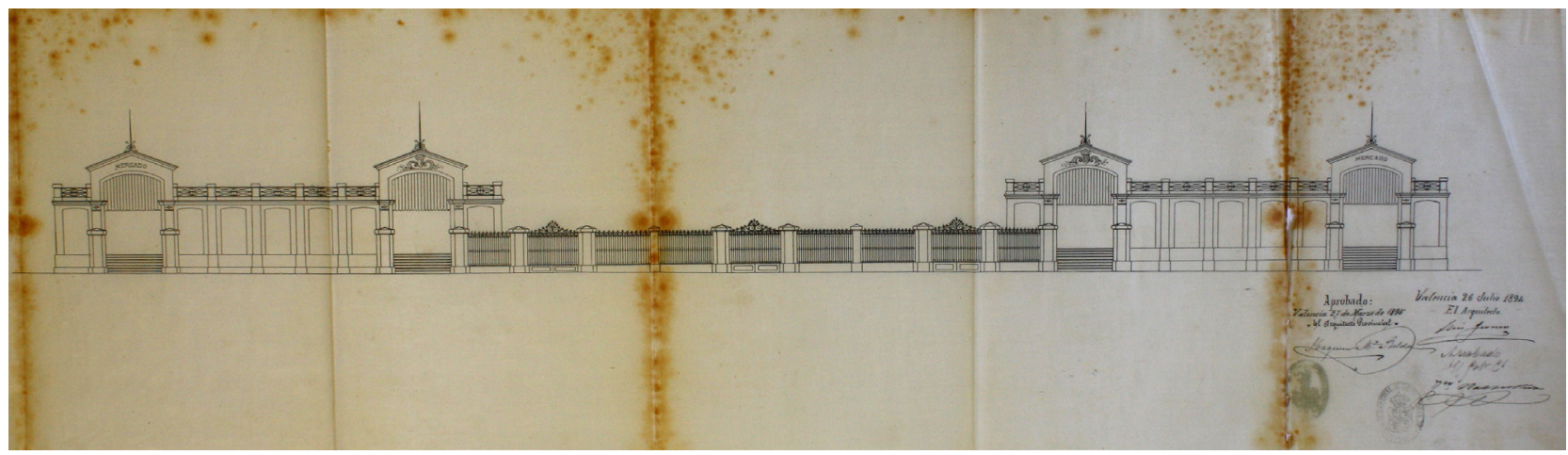

5. Luis Ferreres Soler, Proyecto de construcción del Mercado de Cullera, 1894, alzado de las secciones 1 y 2

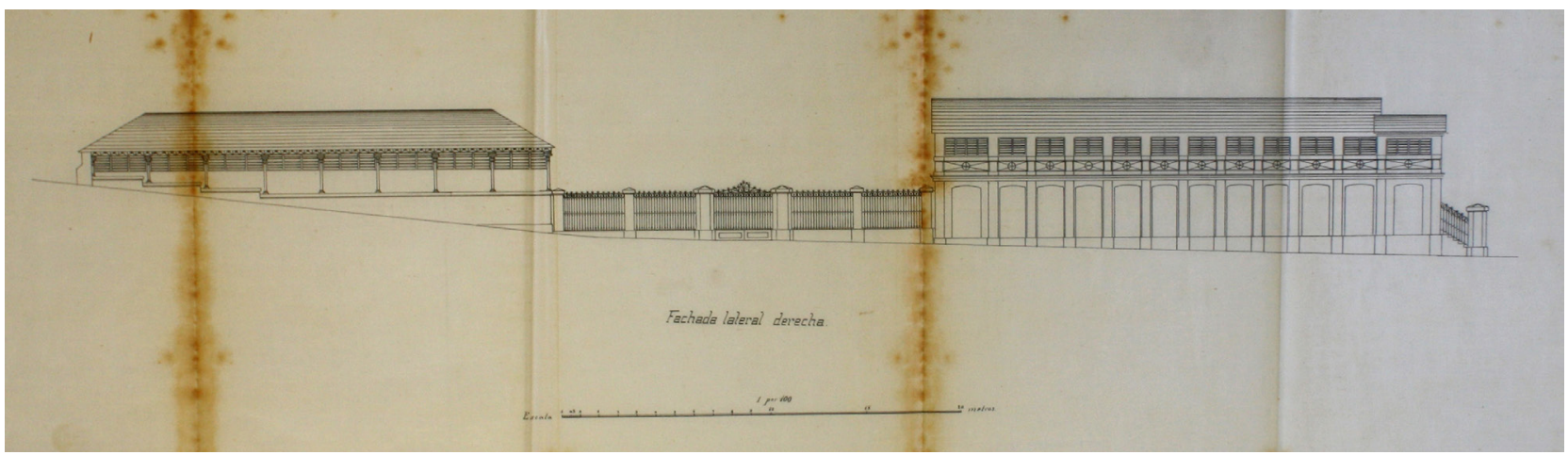

6. Luis Ferreres Soler, Proyecto de construcción del Mercado de Cullera, 1894, alzado de la fachada lateral, secciones 1 y 4

tre ellos por una verja de hierro forjado, cuyos tramos se sustentaran con pilares de fábrica de ladrillo [4]. Ferreres lo concibe como un conjunto cerrado en atención a su mejor mantenimiento. Las entradas desde el exterior se abren sobre los cuatro extremos de las avenidas. Dispone una puerta de tres metros de luz en cada uno de ellos, excepto en el recayente a la calle del Mar donde se abren tres. El mayor número de huecos enfatiza también el carácter principal de esta fachada, al tratarse de la vía más importante y con más afluencia de público de las que rodean el mercado,

El proyecto plantea una jerarquía entre los pabellones de las dos primeras secciones, cuyas fachadas y accesos principales recaen a la calle del Mar, y las posteriores, a las que se accede desde la avenida transversal interior, que se materializa en su arquitectura. Según explica Ferreres «los dos primeros dichos espacios recayentes a la calle del Mar son desde luego los más importantes y por lo mismo en ellos han de colocarse también los puestos de mayor importancia, o sean las lonjas o casetas para la venta de carnes, quesos, ultramarinos y sus similares" ${ }^{9}$. Estas se alinean en tres de los cuatro lados que forman en perímetro de las dos primeras secciones adoptando una planta en forma de $\mathrm{U}$, cuyo lado abierto recae a la calle transversal interior. Las casetas miden tres metros de profundidad por dos de anchura, y se cierran con persianas metálicas enrollables, por lo que sus vendedores podrían dejar en ellos sus mercancías. Esta estructura conforma las fachadas exteriores de ladrillo visto, que se articulan por una sucesión de paneles rehundidos rematados por arcos rebajados, que se repiten de forma modular y se separan por pilastras de fuste liso. Los dos accesos con que cuenta cada uno de los pabellones se enfatizan con un remate triangular a manera de frontón clásico. Como es habitual en algunos equipamientos públicos proyectados por Ferreres, utiliza la fábrica de ladrillo de acuerdo 


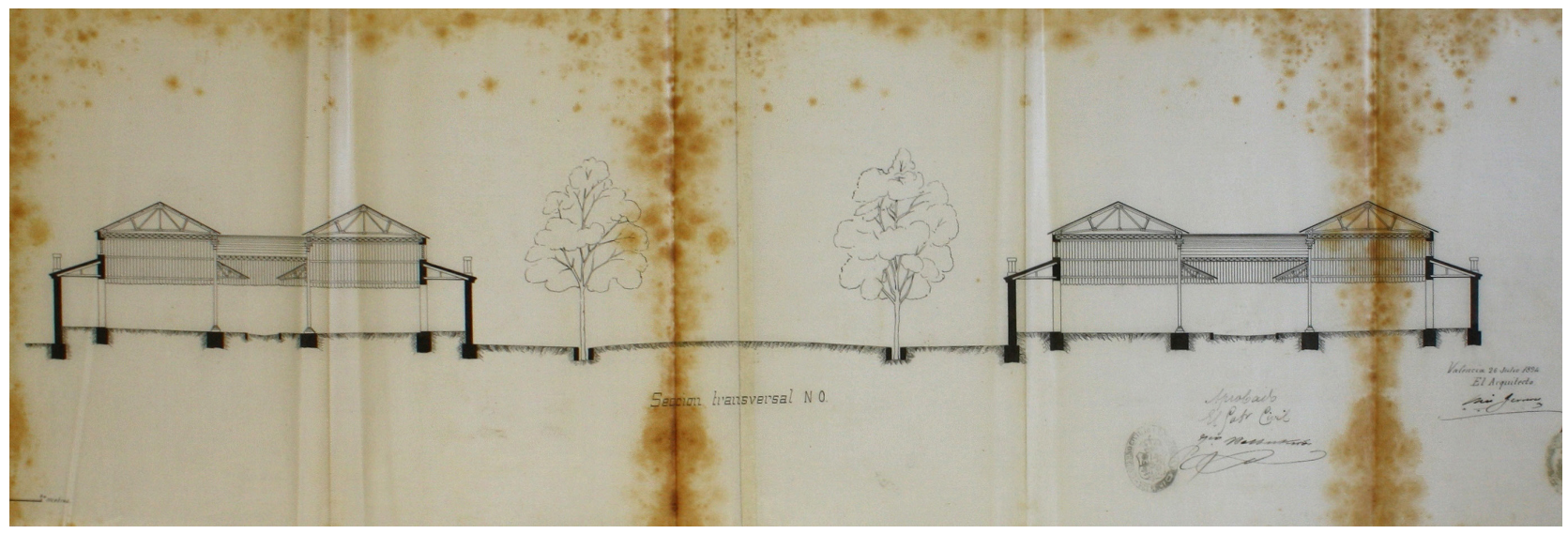

7. Luis Ferreres Soler, Proyecto de construcción del Mercado de Cullera, 1894, sección de los pabellones 1 y 2

con los principios de sinceridad y racionalidad, propios de la arquitectura industrial (Aguilar, 1990: 104), que en cierta manera evocan los valores estéticos de la arquitectura clásica que caracterizaron buena parte de su producción arquitectónica [5], [6]. Los cuerpos que recaen a la calle del Mar se cubren en forma de terraza practicable, a la que se accederán por una escalera situada en el interior de cada una de las secciones, «al objeto de que en los días de determinadas festividades puedan colocarse en ellas sillas que alquilar al público, obteniendo así un nuevo arbitrio en beneficio del Ayuntamiento ${ }^{10}$. En paralelo a los dos ejes longitudinales se adosan sendas naves de 6,5 m, que se unen entre sí en sus extremos por unas crujías, también cubiertas, de 3,5 y $4 \mathrm{~m}$ de anchura respectivamente, donde se sitúan los puestos de venta, quedando como resultado un patio central abierto. En ellos se proyectan unas marquesinas de 1,8 $\mathrm{m}$ adosadas a las columnas de las naves para proteger del sol y de la lluvia a los compradores [7].

Estos dos pabellones ofrecen cierto interés al materializar un estadio de transición entre el tipo de mercado abierto y el cerrado por una caja de fábrica. Aunque en otras regiones españolas este último comenzó a difundirse a principios del siglo XX, en el ámbito valenciano no se generalizó hasta ya entrada la década de los años veinte del pasado siglo con la construcción de los mercados de Villanueva de Castellón (1926), Benifaió (1928) o Carcaixent (1934). Los puestos de venta situados bajo un pórtico cubierto adosado sobre el perímetro de un espacio abierto dedicado al comercio fue una solución habitual en el ámbito valenciano durante el siglo XIX, donde podemos señalar como ejemplo significativo el precedente del actual Mercado Central de Valencia ${ }^{11}$, construido en 1839, que Aguilar (1990: 151) relaciona con los mercados clásicos italianos, donde se combinan el comercio al aire libre con los pórticos o logias donde se sitúan otros puestos de venta, o con una adaptación a una escala más reducida de las propuestas de F. Blondel. El proyecto de Ferreres plantea la cubrición del espacio central con naves paralelas de estructura metálica, formando espacios intermedios abiertos. En un estadio evolutivo posterior del tipo, la superficie central se cubre por una sola nave que alcanza toda la luz y, por tanto, ha de ganar en altura para mantener unas adecuadas proporciones. Hablamos ya de la consolidación del tipo de mercado cerrado por una caja de fábrica, que adopta un carácter marcadamente representativo como monumento urbano del que carece en la etapa anterior.

El alzado de las dos secciones posteriores está formado por una nave de seis metros de luz que recorre los cuatro lados de su perímetro. La sección tercera, de mayor anchura, se halla a su vez cruzada por otra nave [8]. De esta manera se conforman en su interior uno y dos patios abiertos respectivamente, que sirven «para la luz y ventilación interior y al propio tiempo son aumentos de área utilizable para puestos de frutas durante el verano si las circunstancias lo requieren, porque en dicha época no son de temer las inclemencias atmosféricas ${ }^{12}$. Los puestos de venta se alinean a ambos lados de cada nave. En tres de sus caras, excepto en la recayente a la calle transversal, las fachadas son de fábrica de ladrillo visto, ya que a su vez actúan de muro de 


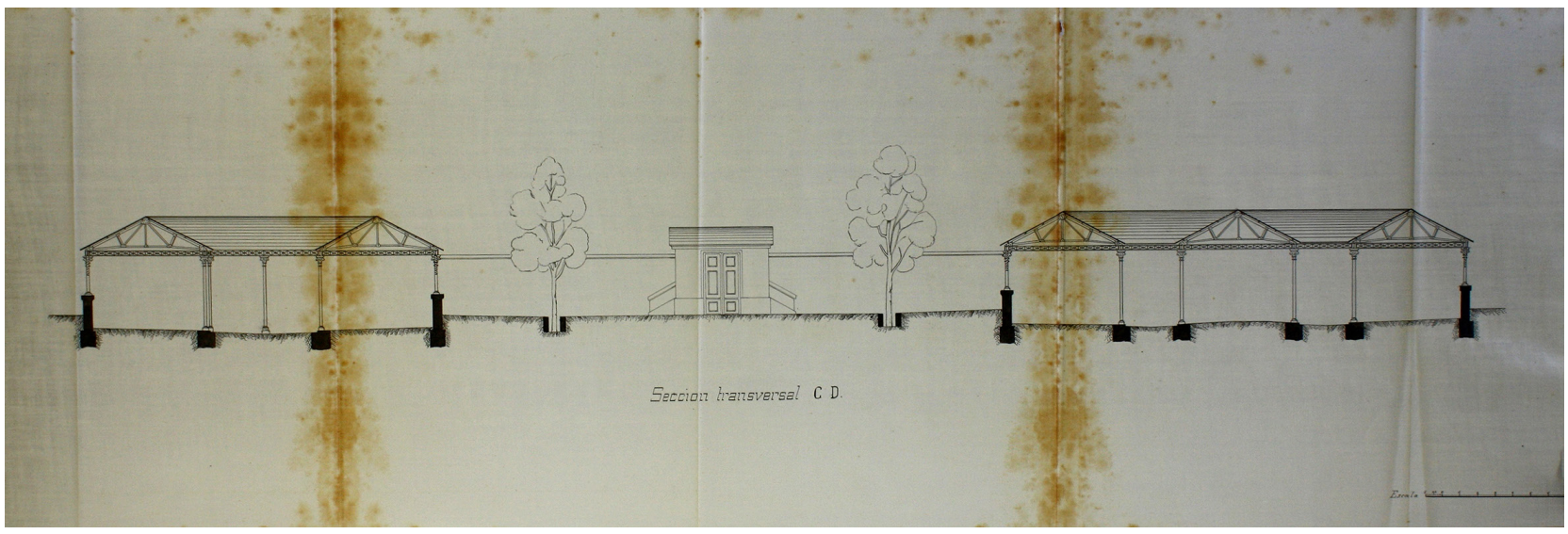

8. Luis Ferreres Soler, Proyecto de construcción del Mercado de Cullera, 1894, sección de los pabellones 3 y 4

contención del desnivel que se forma con las calles exteriores. Por ello las cubiertas apean en los muros laterales sobre cortas columnas de fundición, cuyo espacio sirve a su vez para ventilación interior. A estas secciones se accede desde la calle transversal interior [9].

Las puertas de los diferentes pabellones se sitúan tomando como referencia la orientación de las naves longitudinales que los conforman. El desnivel entre la plataforma del mercado y la cota exterior se salva con unas gradas de piedra de sillería blanca. La entrada a las dos primeras secciones se efectúa por dos vanos de 3,40 m de anchura, cerrados por rejas, con remate inclinado sobre el que se dispone el escudo de la ciudad y el rótulo de la sección, lo que enfatiza su carácter principal. Estas puertas confrontan con otros dos accesos al final de cada una de las naves, que recaen a la avenida transversal interior. La sección tercera dispone de tres entradas y la cuarta de dos, de tres metros de anchura cada una de ellas.
9. Francisco Roglá López, Mercado Municipal de Cullera, 1925, vista del pabellón de la sección tercera, desde el eje transversal, Biblioteca Valenciana Nicolau Primitiu. Fons Roglá

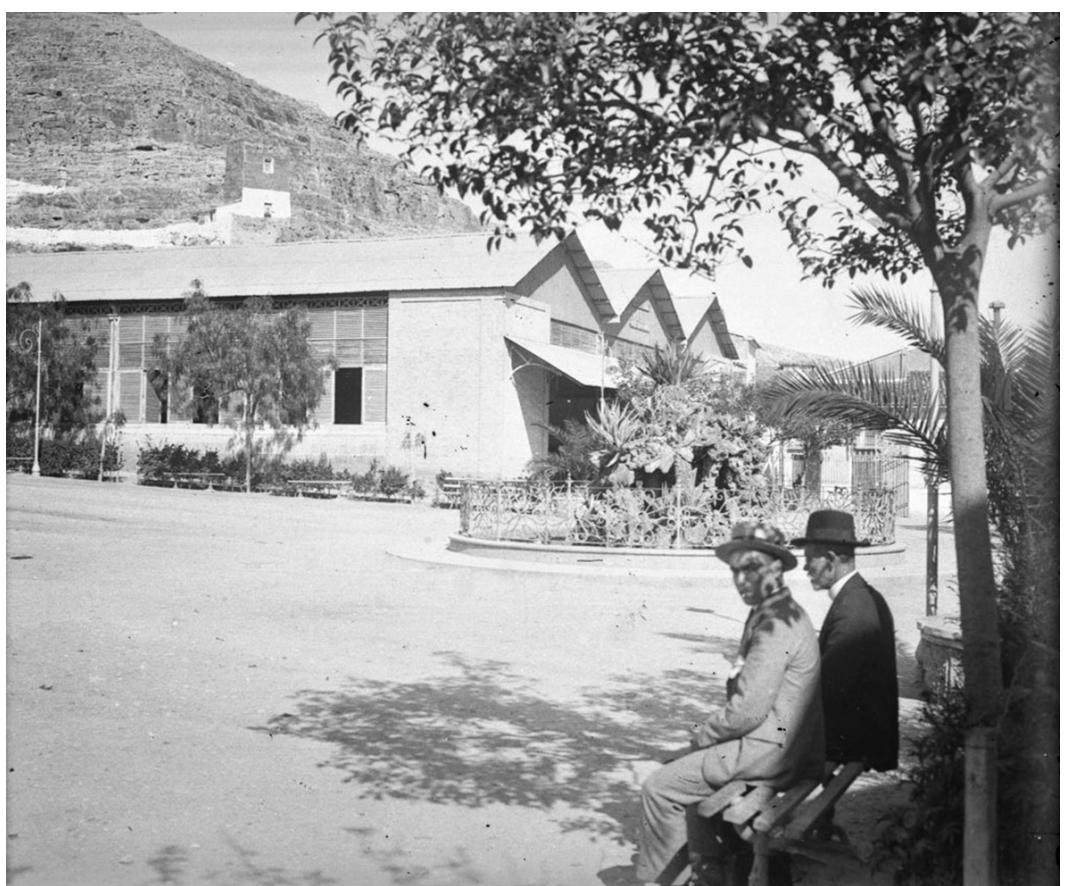




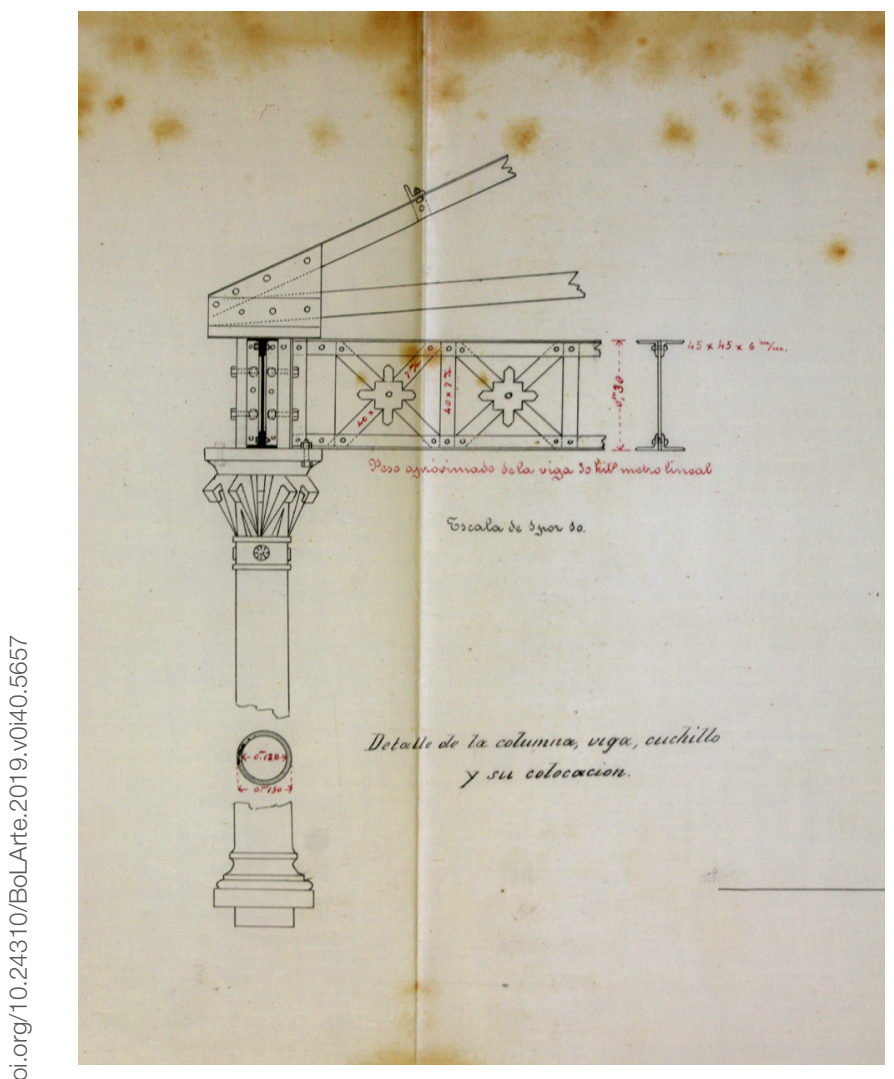

10. Luis Ferreres Soler, Proyecto de construcción del Mercado de Cullera, 1894, detalle del apeo de las armaduras de la cubierta

De acuerdo con el texto de la memoria se deduce que Ferreres, una vez definida la planta del edificio, planteó sobre el plano un reparto de puestos, del cual resultó un espacio recorrible, que a su vez sirvió como base para definir los ingresos a los pabellones. Se proyectan 56 lonjas: 29 para la primera sección y 27 para la segunda. Proyecta los puestos para la venta de frutas y verduras de unas dimensiones aproximadas de 1,5 x 1,5 m aproximadamente, con cuya superficie se obtiene un total de 264 puestos, repartidos de la siguiente manera: 49 en la primera sección, 41 en la segunda, 101 en la tercera y 73 en la cuarta. Sumando los puestos y las lonjas arroja un total de 320 puntos de venta, que se consideran suficientes para las necesidades de la población, pues en ese momento el mercado estaba conformado por 306 puestos, pero de unas dimensiones menores que oscilaban alrededor del metro cuadrado. En base a la anchura de las naves longitudinales los espacios libres para la circulación serían de 3,4 m para las calles interiores de las dos primeras secciones y de $3 \mathrm{~m}$ para las dos últimas. De acuerdo con el alzado proyectado, todos los edificios pueden recorrerse completamente a cubierto.

A pesar de que desde el exterior predomina la materialidad de la fábrica de ladrillo que cierra los pabellones que lo componen, según explica el mismo Ferreres, «el elemento principal que se propone para la construcción del mercado proyectado es el hierro. Columnas de fundición embrochaladas por vigas de celosía, colocadas sobre los capiteles, sirven de soporte a las formas o cuchillos de armadura destinados al sostenimiento de las planchas de hierro onduladas y galvanizadas que han de cubrir las naves» ${ }^{13}$. En este sentido se trata de un proyecto atípico en relación con los mercados que en ese momento se proyectaban y se construian en el área de Valencia, pues se recurre a estructuras abiertas formadas por cubiertas con armaduras metálicas sostenidas por columnas de fundición, que generalmente se articulaban en paralelo o en forma de cruz. Esta segunda opción es la que Ferreres eligió para sus mercados de Algemesí, Moncada y Silla. Como justifica en la memoria, los condicionantes de una parcela en desnivel y la necesidad de disponer de tiendas cerradas para la venta de carnes, salazones y ultramarinos le llevaron a plantear edificios cerrados. Igual que hizo su obra más representativa, el matadero de Valencia, combina la arquitectura de hierro de sus estructuras interiores con los cerramientos perimetrales de ladrillo visto. En todos los pabellones los cuchillos apean sobre las vigas de celosía, a las que se sujetan con aletas unidas con pernos [10]. En las secciones 1 y 2 estas vigas descansan en su perímetro exterior sobre pilares de ladrillo y en su interior sobre los capiteles de las columnas de fundición que sostienen a su vez las ménsulas de hierro forjado que conforman las marquesinas. En las secciones 3 y 4 estas se asientan en todos los casos sobre los capiteles de las columnas de fundición ${ }^{14}$.

A finales del siglo XIX eran muy pocas las poblaciones valencianas que disponían de una red de canalización de agua potable. Por ello, en muchos proyectos de mercado no se planteaba el tema de la disponibilidad de agua para la limpieza. Ferreres muestra una especial sensibilidad por la higiene en los equipamientos públicos. En el matadero de Valencia diseñó un sistema de limpieza por inundación, considerando la escasa presión que en ese momento tenía la red de abastecimiento municipal (Aguilar, 1990: 164). Cullera no disponía en aquel momento de una red pública, por lo 
que propone la excavación de un pozo, considerando la presencia de mantos freáticos a escasa profundidad. El agua sería elevada a un depósito por una bomba aspirante-impelente, y de allí se conduciría a una fuente situada en cada una de las secciones por medio de tuberías cerámicas y metálicas. El pozo y el depósito se situarán en la caseta que se proyecta al efecto al fondo de la avenida de longitudinal, junto a la escalera que salva el desnivel con la calle.

Otro aspecto relacionado con la higiene es el de los pavimentos. Las soluciones propuestas son habituales en los mercados valencianos del momento donde se adoptan los materiales propios de la arquitectura vernácula. El área situada bajo las naves destinada a vendedores se pavimentará con adoquines de piedra arenisca de rodeno. Los suelos de patios o espacios abiertos de cada una de las secciones se revestirán con cantos rodados que forman paneles con diferentes dibujos enmarcados por un encintado de ladrillo dispuesto a panderete.

\section{Conclusión}

El proyecto estudiado se enmarca de forma coherente en la trayectoria profesional de Luis Ferreres y se sitúa en una de las épocas más fecundas de su actividad. No recurre a las propuestas habituales para las ciudades medianas, basadas en edificios abiertos de una nave, de naves adosadas en paralelo o dispuestas en forma de cruz, que él mismo también utilizaría en otros mercados. Este proyecto es fruto de una concienzuda reflexión donde contempla las diversas necesidades actuales y futuras a las que el conjunto construido debe responder, por lo que la obra resultante trasciende el hecho meramente arquitectónico y se integra a nivel formal y funcional en el nuevo modelo de ciudad moderna para el que se concibe. Una prueba de ello es que, un siglo más tarde, aunque con algunas modificaciones y adaptaciones, continúa satisfaciendo las mismas necesidades para las que fue proyectado. La prioridad otorgada a la función sobre la forma, característica en su obra, le lleva a plantear una propuesta tipológica singular, adaptada al lugar, que guarda estrechos paralelismos con otras realizaciones y proyectos que se organizan a partir de diversos ejes cubiertos, que en este caso concibe como amplios espacios abiertos, que parten del concepto que Víctor Baltard introdujo en las Halles Centrales de París.

El proyecto es el resultado de la combinación de tres modelos de mercado. El tinglado abierto, al que responden básicamente los pabellones de las secciones tres y cuatro, el mercado cerrado cubierto con estructura metálica y rodeado por una caja de fábrica, en el que se inspiran las secciones una y dos, y el mercado al aire libre o plaza, al que destinaría el espacio de las dos amplias avenidas transversales durante el verano, cuando aumentaba la venta de productos agrícolas, o donde se ubicaría el mercado semanal. Pero la principal novedad respecto a la mayoría de mercados construidos, donde el comercio ambulante ocupaba las calles adyacentes, es que en Cullera esta actividad no se desarrollaría en plena vía pública, sino que vendría a realizarse en un amplio espacio interior abierto, especialmente acondicionado y dedicado a este uso.

La estética de su arquitectura se basa en la sinceridad en la utilización de los materiales, impuesta por funcionalidad de la obra, donde recurre a cuidadas fábricas de ladrillo visto moduladas con formas sencillas como pilastras o paneles rehundidos, y a la utilización del hierro como material visto. Por ello, a pesar de su aparente sencillez, relacionada con la discreción que caracterizó la personalidad de su arquitecto, podemos valorar el mercado de Cullera como un edificio singular de gran interés tipológico en el panorama arquitectónico valenciano, en el que confluyen diversos referentes con el fin de ofrecer una solución audaz y creativa a los condicionantes planteados.

\section{Notas}

1 El pliego de condiciones se publicó en la Gazeta de Madrid, 3 de octubre de 1899, n. ${ }^{3}$ 376, pp. 34-36, y en la Revista de obras Públicas, 1899, n. ${ }^{\circ} 224$, pp. 379-381.

2 Arxiu Històric de Cullera (AHC), 9.10. L-1. Proyecto de construcción de un nuevo mercado. Este expediente contiene el proyecto arquitectónico íntegro formado por memoria, pliego de condiciones y planos, y la documentación administrativa de todo el proceso de contratación y ejecución de las obras.

3 Puede encontrarse un estudio del mismo en Aguilar (1990: 161-167). 
4 El mercado de Algemesí ha sido estudiado por Doménech (1988), Ferrer (1997) y Besó (2017). El de Moncada fue publicado por Ferrer (2000), quien no atribuye autoría al no disponer del proyecto arquitectónico, como lo hace con posterioridad Taberner (2009: 141). Y el de Silla se aborda en el trabajo de Ferrer (1997) ya citado.

5 La previsión de modernización del puerto de Cullera, unido al desarrollo de una agricultura especializada en el cultivo del arroz sobre las tierras de marial situadas junto a la desembocadura del Júcar, hizo que se plantearan nuevas posibilidades de crecimiento y desarrollo para la ciudad, que se materializaron en la construcción de la línea Silla-Cullera en 1878, que enlazaba con el ferrocarril de la Compañía del Norte, y en la confluencia varias carreteras de segundo y tercer orden que buscaban la estación y el puerto como vías de salida a las producciones agrícolas de las poblaciones cercanas.

6 Arxiu Històric Municipal de Sueca, Urbanismo, caja 23, exp. 3. El proyecto fue redactado por el arquitecto Antonio Monforte el 30-10-1877. Para conocer más detalles de este edificio ver Besó (2017).

7 AHC, 9.10. L-1. Proyecto de construcción de un nuevo mercado, memoria, fol. 9 r. Según Ferreres, «porque la construcción por partes o secciones facilita extraordinariamente la ejecución, es por lo que resulta conveniente estudiar una disposición general [...] para que cada sección forme la unidad de una composición que aisladamente tenga la forma y condiciones adecuadas al objeto de su destino y que en su conjunto las complete y armonice [...]. Si el proyecto hubiera de realizarse por secciones, cada una forma una construcción independiente que por sí misma se determina y responde a las necesidades que la informan, y si el proyecto se realiza en conjunto o de una sola vez, entonces resultan partes de un mismo todo que igualmente y con más razón han de satisfacer al propio intento". Como ejemplo de los inconvenientes que ocasiona la demora en la ejecución de un proyecto unitario, cita el ya referido mercado de la vecina localidad de Sueca, que en aquel momento que se construyó en cinco fases conforme se fueron realizando las expropiaciones necesarias.

8 Aunque no lo indica de forma explícita en la memoria, posiblemente el carácter abierto con el que plantea los nuevos edificios, que contrasta con los pabe\|lones cerrados que proyecta, posiblemente guarde relación con la necesidad de no disminuir la capacidad para la concentración de público el día de la fiesta de la Virgen, pues de esta manera podrían continuar disponiendo de la totalidad del nuevo espacio cubierto.

9 AHC, 9.10. L-1. Proyecto de construcción de un nuevo mercado, memoria, fol. 16 r.

10 AHC, 9.10. L-1. Proyecto de construcción de un nuevo mercado, memoria, fol. 17 r.

11 El antiguo mercado de Alzira también respondía a este tipo. Allí, el espacio central abierto fue ocupado parcialmente por un pabellón abierto de naves paralelas sustentadas por columnas de fundición, proyectado por el arquitecto Antonio Martorell en 1882 (Doménech, 1988: 280; Besó, 2017 : 344 y 361).

12 AHC, 9.10. L-1. Proyecto de construcción de un nuevo mercado, memoria, fol. 17 vo.

13 AHC, 9.10. L-1. Proyecto de construcción de un nuevo mercado, memoria, fol. 20 vo. Ferreres fue un valedor de la introducción del hierro en la arquitectura, pues como hemos indicado, inició su andadura profesional con Francisco Jareño en las obras de la Biblioteca Nacional de Madrid, cuyo depósito de libros, hoy desaparecido, resolvía enteramente con piezas metálicas. Además destacó por la defensa del uso del hierro en la arquitectura en algunos de sus escritos. Lo utiliza en el proyecto de 1883 del nuevo mercado central de Valencia, y es el material único en el resto de mercados que proyecta (Taberner, 2009: 133).

14 Durante la ejecución del proyecto se realizaron algunos cambios respecto al proyecto inicial. Se eliminaron los espacios interiores abiertos que se planteaban en el proyecto, por lo que todos los pabellones quedaron cubiertos con tres naves paralelas. Los intercolumnios de las secciones posteriores se cerraron completamente con láminas inclinadas, que en el proyecto solamente figuraban en el tercio superior. Las viseras de madera previstas en las fachadas de los cuatro pabellones recayentes a la calle transversal con la finalidad de proteger el interior de los efectos del sol, fueron sustituidas por marquesinas metálicas. Este hecho se refleja en las primeras fotografías conservadas del conjunto, inmediatamente posteriores a su construcción, y explicaría también la diferencia respecto al presupuesto de contrata, pues el coste de la obra ascendió a un total de 152.231,7 ptas.

15 Entre 1998 y 2009 se realizó una rehabilitación integral del conjunto. Las secciones 1 y 2, que continúan manteniendo su función comercial, fueron rehabilitadas para adaptarlas a la normativa vigente. Aunque conservan la estructura metálica, esta convive con los conductos de aire acondicionado, bajantes y otras instalaciones, que al estar pintadas del mismo color dificulta una adecuada apreciación. Los testeros que recaen al espacio interior se han cerrado con un muro pantalla de vidrio para mantener la imagen abierta con que se construyeron y poder alcanzar dentro las condiciones adecuadas de climatización. Las secciones 3 y 4 conservan su aspecto exterior, mientras que su interior se ha transformado para adaptarlo a nuevos usos como auditorio y sala polivalente respectivamente. En 1938, durante la Guerra Civil se construyó bajo estas dos secciones un refugio antiaéreo subterráneo, que ha sido restaurado y abierto al público recientemente.

\section{Bibliografía}

AGUILAR CIVERA, Inmaculada (1990), El orden industrial en la ciudad. Valencia en la segunda mitad del siglo XIX, Diputació de Valencia, Valencia.

- (1998), Arquitectura industrial: concepto, método y fuentes, Museu d’Etnologia de la Diputació de València, Valencia. ARCINIEGA GARCÍA, Luis (2002), «Las murallas de Cullera», en FURIÓ, Antoni y APARICI, Josep (eds.), Castells, torres i fortificacions en la Ribera del Xúquer, Universitat de València, València, pp. 231-244.

BENITO GOERLICH, Daniel (1992), La arquitectura del eclecticismo en Valencia. Vertientes de la arquitectura valenciana entre 1875 y 1925, Ajuntament de València, Valencia.

BESÓ ROS, Adrià (2017), «Abastecer la ciudad moderna. Mercados de hierro en la comarca de la Ribera del Júcar (1877-1904)», Artigrama, n. ${ }^{\circ} 32$, pp.335-366.

CASTAÑER MUÑOZ, Esteban (2006), La arquitectura del hierro en España. Los mercados del siglo XIX, Real Academia de Ingeniería, Madrid.

CLAIRAC Y SAENZ, Pelayo (1877), Diccionario general de arquitectura e ingeniería, Zaragozano y Jaime, Madrid, 5 vols. 
DOMÉNECH ALCOVER, Eduardo (1988), «Arquitectura del hierro en la Ribera Alta: Los mercados de Alzira, Algemesí y Alberic», Al-Gezira, Revista d'Estudis Històrics-Ribera Alta, n. ${ }^{4} 4-5$, pp. 273-313.

FERRER SELMA, Amparo (1997), «Los mercados de Algemesí y Silla», Torrens, n. 11, pp. 141-190.

- (2000), «Els mercats de Burjassot, Montcada i Godella», en FRECHINA, Josep Vicent (coord.), Actes del I Congrés d'Estudis de l'Horta Nord, Centre d'Estudis de l'Horta Nord, València, pp. 419-446.

GIRALT CASADESÚS, R. (1937), Mercados. Teoría y práctica de su construcción y funcionamiento, Cuerpo de Arquitectos Municipales de España, Barcelona.

HIDALGO DELGADO, Francisco (2012), «De lo proyectado a lo construido. El Mercado Central de Valencia», EGA Expresión Gráfica Arquitectónica, n. ${ }^{\circ}$ 19, pp. 114-125.

TABERNER PASTOR, Francisco (1988), Luis Ferreres Soler, arquitecto, Dirección General para la Vivienda y Arquitectura, Madrid.

- (2009), «Trayectoria profesional del arquitecto Luis Ferreres Soler (1852-1926): Entre la audacia y la discreción», en MARTÍ, Javier, GARCÍA, Marta y CASAO, Rosario (coords.), Otra lectura de la reforma interior: en torno al proyecto de Luis Ferreres: catálogo de la exposición, Ajuntament de València, València, pp. 131-145. 